\title{
\begin{tabular}{l|l} 
pcori $)$ & PATIENT-CENTERED OUTCOMES RESEARCH INSTITUTE \\
RESEARCH SUMMARY
\end{tabular}
}

\section{Can People Who Have Experience with Serious Mental Illness Help Peers Manage Their Health Care?}

Principal investigator

John Sinclair Brekke, PhD, MS
Organization

University of Southern California

\section{What was the research about?}

Serious mental illnesses, such as bipolar disorder, schizophrenia, or major depression, can affect a person's mood, thinking, and behavior. These illnesses often last a long time and can disrupt people's lives. People with serious mental illness die 10 to 30 years younger than people who don't have mental illness, often from health problems that could have been treated or prevented. People with serious mental illness may find it hard to find doctors who understand them. They may also find it hard to follow plans for preventing or treating diseases.

The research team wanted to help people with serious mental illness better manage their health and health care. To do so, the team used a peer navigator program. A peer navigator is someone who has experience with mental illness. Peer navigators help people with serious mental illness get the care they need. The research team compared patients in the peer navigator program with those who received usual mental health care.

\section{What were the results?}

The research team found that patients in the program started to see their regular doctors more often, were more satisfied with their doctors, and preferred outpatient care over urgent care more than those who weren't in the program.
Patients in the program also had less severe pain learned more about health problems they already had, and became more confident that they could manage their health care than those who weren't in the program.

Compared with usual care, the program didn't improve patients' mental health, social skills, or health habits.

\section{Who was in the study?}

The study included 151 patients with serious mental illness who received care at a large mental health center in California. The average patient age was 47, and 54 percent of patients were women. Sixty percent of patients in the study were Hispanic or Latino, 25 percent were white, 8 percent were African American, and 8 percent were other or mixed race.

\section{What did the research team do?}

The research team compared two groups of patients. Both received usual mental health care. The research team assigned about half of the patients by chance to the peer navigator program. These patients received peer navigation services for six months. They worked with peer navigators to make a healthcare plan and set health goals. Peer navigators also helped patients set appointments, went with patients to see their doctors, and coached patients on skills for managing health care. 


\section{What were the limits of the study?}

The study took place in a single mental health center in California. The results might be different in other places. Also, the study was not large enough to see if the program worked differently for certain groups of people, such as for women or men.

Future research could see if certain parts of the program are more helpful than other parts.

\section{How can people use the results?}

Clinics that treat people with serious mental illness could consider offering a peer navigation program like the one in this study.

To learn more about this project, visit pcori.org/Brekke067. 